in the shop and right-to-know training, emergency planning, ergonomics, personal protective equipment, respiratory protection, paint booth and mixing room, electrical and machine safety). Facilities that were working or had worked with a safety consultant had significantly fewer missing items at baseline $(\mathrm{p}<$ 0.03 ), but not at follow-up. On average, shop owners chose to correct $59 \%$ of the missing items $(\mathrm{SD}=17 \%)$ and after one year reported a completion rate of $70 \%(\mathrm{SD}=28 \%)$. One-year assessments indicate that, on average $56 \%$ of the items selected for improvement were actually completed $(S D=27 \%)$.

Conclusions Results indicate that most business owners were able to improve health and safety in the shop if they were provided specific information about hazards and solutions, received regular reminders and utilised tailored technical assistance.

\section{DOES FEEDBACK ON MEASURED CONCENTRATIONS REDUCE THE LEVEL OF EXPOSURE TO DUST AMONG FARMERS?}

1' Basinas, 'Sigsgaard, ${ }^{2}$ Kromhout, 'Bønløkke, 'Schlünssen. 'Department of Public Health, Aarhus University, Aarhus, Denmark; ${ }^{2}$ Institute for Risk Assessment Sciences (IRAS), Utrecht University, Utrecht, The Netherlands

\subsection{6/oemed-2013-101717.259}

Objective To assess whether exposure can be reduced by providing feedback to the farmers concerning the levels of dust that they are daily exposed to in their farm.

Methods The personal dust levels of farmers in 53 pig and 25 dairy cattle farms were evaluated in 2 measurement series performed approximately 6 months apart. Detailed information on technical parameters and farm characteristics were also registered. Participating farms were a priory randomly divided into a control $(n=39)$ and an intervention group $(n=39)$. Shortly after the first visit, farm owners in the intervention group received a letter with information on the measured dust concentrations on their farm together with some general advises on exposure reduction strategies (e.g. use of respirators during certain tasks). Relationships between measured dust concentrations and intervention status were quantified by means of linear mixed effect analysis, with farm id as a random effect. Season, type of farming, visit, intervention status and their two-way interactions were tested as fixed effects.

Results After adjustment for season and farm type we found no effect by intervention status. There was no interaction by type of farm, but measured dust levels on the second visit were significantly lower than during the first visit. Similar results were observed in models stratified by type of farming, where the effects of visit were most clearly observed among pig farms.

Conclusion These preliminary findings suggest no interventional effects on the levels of exposure; though, the presence of the investigation itself seems to reduce the levels of exposure. By June 2013, the authors intend to present the above results along with those from further analysis addressing potential changes in working patterns and hygienic parameters during the second exposure evaluation.

\section{INTERVENTIONS TO PREVENT OCCUPATIONAL NOISE- INDUCED HEARING LOSS - A COCHRANE REVIEW}

${ }^{1} \mathrm{~V} J$ Verbeek, ${ }^{2}$ Kateman, ${ }^{3}$ Morata, ${ }^{4}$ Dreschler, ${ }^{5}$ Mischke. ${ }^{1}$ Kuopio, Finland; ${ }^{2}$ Center of Expertise in Hearing and Noise Problems, Doetinchem, Nederland; ${ }^{3}$ National Institute for Occupational Safety and Health (NIOSH), Cincinnati, United States of America;
${ }^{4}$ Academic Medical Centre, Amsterdam, Nederland; ${ }^{5}$ Cochrane Occupational Safety and Health Review Group, FIOH, Kuopio, Finland

\subsection{6/oemed-2013-101717.260}

Objectives Millions of workers worldwide are exposed to noise levels that increase their risk of hearing impairment. Little is known about the effectiveness of hearing loss prevention interventions. Therefore we assessed the effectiveness of non-pharmaceutical interventions for preventing occupational noise exposure or occupational hearing loss compared to no intervention or alternative interventions.

Methods We followed the methods prescribed by the Cochrane Collaboration. We searched 8 different electronic databases to 25 January 2012. We included randomised controlled trials, controlled before-after studies and interrupted time-series of nonclinical hearing loss prevention interventions among workers. Two authors independently assessed study eligibility and risk of bias and extracted data. We combined similar studies in a metaanalysis using RevMan5 and assessed statistical heterogeneity with the $\mathrm{I}^{2}$ statistic.

Results We included 25 studies. We found no controlled studies on engineering controls for noise exposure but one interrupted time-series on legislation to reduce noise exposure. Eight studies evaluated effects of personal hearing protection devices. Sixteen studies evaluated hearing loss prevention programs (HLPPs). The implementation of stricter legislation was shown to reduce noise levels in workplaces. Case studies showed that substantial reductions in noise levels can be achieved, but there are no controlled studies of the effectiveness of such measures. Better use of hearing protection devices as part of HLPPs reduces the risk of hearing loss, whereas for other program components of HLPPs we did not find such an effect. The overall quality of studies was low to very low.

Conclusions There is still a considerable risk of hearing loss in workers that are considered to be protected by hearing loss prevention programs. The effectiveness of hearing protection devices depends on training and their proper use. Better implementation and reinforcement of hearing loss prevention programs is needed. Better evaluations of technical interventions and long-term effects are needed.

\section{WHEN DOES RESEARCH SERVE PREVENTION?}

Dr Wergeland. Labour Inspection Authority, Oslo, Norway

\subsection{6/oemed-2013-101717.261}

Objectives It is commonly considered that prevention should be based on scientific evidence. But the relationship between research and prevention is not a simple one. What constitutes evidence, and how should results be interpreted? Research may sometimes delay prevention. The aim of this study is to identify factors that have influenced the impact of research on prevention in Norway over the last century.

Methods We examined available documents about two large industries, the production of silicon carbide, established in 1913, and the production of primary aluminium, established in 1908 . Research papers on occupational exposure and workers health in these industries have been examined, together with recommendations issued by members of the scientific community. "State of the art" scientific knowledge has been compared with concurrent initiatives taken by various stakeholders, including the Norwegian Labour Inspection. 
Results Methodologically simple research papers identified targets for disease prevention early on in both industries: from 1918 for silicon carbide, and from 1936 for primary aluminium. Later and more complicated studies of disease mechanisms, and studies involving detailed exposure characterisations, do not seem to have served preventive practice to any great extent. The scientific community tends to support stakeholders request for more research before lowering of TLVs or reducing exposure. Disagreement about what constitutes evidence has delayed prevention and stimulated research, but the research questions were not always relevant for prevention. The Norwegian regulatory model, with environmental standards based on tripartite consensus, may have discouraged technological innovation.

Conclusions Regulatory authorities must accept documentation of harmful exposure as sufficient evidence, long before the scientific community is ready to reject the null hypothesis of no risk. Quasi-experimental prevention can eradicate disease earlier than prevention based on too much evidence. But we may never know exactly why our efforts seemed to work.

\section{Session: 7. Occupational asthma}

\section{\begin{tabular}{|l|l}
262 & SHORT-TERM LUNG FUNCTION EFFECTS AFTER
\end{tabular} OCCUPATIONAL EXPOSURE TO CLEANING PRODUCTS}

${ }^{1} \mathrm{D}$ V Vizcaya, ${ }^{2} \mathrm{M}$ C M Mirabelli, ${ }^{3} \mathrm{D}$ G Gimeno, ${ }^{1} \mathrm{~J} \mathrm{M}$ A Anto, ${ }^{4} \mathrm{G}$ D Delclos, ${ }^{5} \mathrm{M}$ Rivera, 1 J P Z Zock. 'Centre for Research in Environmental Epidemiology (CREAL), Barcelona, Spain; ${ }^{2}$ Wake Forest School of Medicine, Winston-Salem, North Carolina, United States of America; ${ }^{3}$ The University of Texas Health Science Center at Houston, SPH, San Antonio, Texas, United States of America; ${ }^{4}$ The University of Texas School of Public Health, Houston, Texas, United States of America; ${ }^{5}$ University of Montreal Hospital Research Centre (CRCHUM), Montreal, Quebec, Canada

\subsection{6/oemed-2013-101717.262}

Objective To evaluate the acute effects of exposure to cleaning products on lung function of female cleaning workers.

Methods A panel study including 21 female cleaners with persistent asthma symptoms was nested within a case-control study. Participants recorded the use of cleaning products in 2-week diaries resulting in 312 person-days. All participants were trained to perform lung function testing using a PIKO- $1^{\circledR}$ device to measure $\mathrm{FEV}_{1}(\mathrm{~mL})$ and PEF (L/min) three times per day (in the morning after waking-up, at midday and in the evening before going to sleep). Associations between cleaning products and $\mathrm{FEV}_{1}$ and PEF in the evening of the same day of exposure, in the morning next day and $\mathrm{FEV}_{1}$ and PEF's diurnal variation (amplitude over daily mean) were evaluated using linear mixed regression analysis. All models included a random term for individual and were adjusted for age, height, number of cigarettes smoked, respiratory infection, and respiratory medication. The reference category for all comparisons was "No use of cleaning products".

Results Evening $\mathrm{FEV}_{1}$ and PEF were $8.7 \mathrm{ml}$ (95\% confidence interval (CI) 1.7-15.7) and 36.9 1/min (CI 4.3-69.5), lower on days when three or more cleaning sprays were used, respectively (p-values for trend: 0.054 for $\mathrm{FEV}_{1}$ and 0.053 for PEF). Evening $\mathrm{FEV}_{1}$ significantly decreased after exposure to hydrochloric acid $(30.8 \mathrm{ml})$ and solvents $(37.6 \mathrm{ml})$. Diurnal variation in $\mathrm{FEV}_{1}$ increased on days using ammonia (12.7\%), lime-scale removers (9.3\%), air-fresheners (7.2\%) and multiuse products (6.8\%). Diurnal variation in PEF increased on days using ammonia $(17.0 \%)$, lime-scale removers (13.0\%), powder detergents (11.4\%), and air-fresheners (8.6\%). Morning $\mathrm{FEV}_{1}$ decreased on days following the use of solvents $(53.0 ; 36.3-69.6)$, hydrochloric acid (26.3 ml; CI: 14.7-37.9), powder detergents (26.1; 16.7-35.6), and degreasers (19.1; 12.6-25.7).

Conclusions Acute changes in lung function suggest that the use of specific cleaning products may exacerbate pre-existing asthma.

\section{THE RISK OF REDEMPTION OF ASTHMA PHARMACEUTICALS AMONG WELDERS: A NATIONWIDE FOLLOW-UP STUDY}

${ }^{1} \mathrm{P}$ K Kristiansen, ${ }^{2}$ Jørgensen, ${ }^{2}$ Bonde. ${ }^{1}$ Copenhagen NV, Denmark; ${ }^{2}$ Department of Occupational and Environmental Medicin, Bispebjerg Hospital, Copenhagen NV, Denmark

\subsection{6/oemed-2013-101717.263}

Objectives The purpose was to examine if stainless steel and mild steel welding confers an increased risk of bronchial asthma. Methods A Danish national company-based historical cohort of 5,499 ever-welders and 1,514 never-welders was with the Danish Medicinal Product Registry followed from 1995 through 2011 to identify the first-time redemption of asthma pharmaceuticals including Beta-2-adrenoreceptor agonists, Adrenergic and other drugs for obstructive airway diseases, Inhaled Glucocorticoids. Lifetime exposure to welding fume particulates was estimated by combining questionnaire data on welding work with a welding exposure matrix based on more than 1000 personal measurements of ambient air concentrations of welding fume particulates. The estimated exposure accounted for calendar-time, welding intermittence, type of steel, welding methods, local exhaustion and welding in confined spaces. Hazard ratios (HR) with $95 \%$ confidence intervals (CI) were calculated using a Cox proportional hazards model adjusting for potential confounders.

Results The average incidence of redemption of asthma pharmaceuticals in the cohort was $17 / 1000$ years (95\% CI $0.01-0.02$ ). Asthma pharmaceuticals were not redeemed more often among stainless steel $(\mathrm{n}=3874)$ and mild steel welders $(\mathrm{n}=1625)$ than among never-welders. Among ever-welders redemption of asthma pharmaceuticals was not related to life-long exposure to welding fume. Analyses of specific subgroups of asthma pharmaceuticals did not reveal consistent associations with welding exposure. However, among non-smoking stainless steel welders the risk increased with cumulative welding dust exposure (HR for high- vs. low level exposed 1.41, 95\% CI 1.06-1.89).

Conclusions The results showed no consistent association between lifetime exposure to welding fume and use of asthma pharmaceuticals. However, an increased risk of asthma pharmaceuticals among non-smoking stainless welders may indicate that stainless welding does confer an increased risk of asthma, which escapes detection among smokers having a high prevalence of obstructive airway disease.

\section{OCCUPATIONAL ASTHMA IN NEW ZEALAND SAWMILL WORKERS: A LONGITUDINAL STUDY}

'D J Mclean, 'Douwes, ${ }^{1}$ Van Dalen, ${ }^{2}$ Demers, ${ }^{1}$ Cheng, ${ }^{1}$ Shanthakumar, ${ }^{3}$ Pearce. ${ }^{1}$ Massey University, Wellington, New Zealand; ${ }^{2}$ Occupational Cancer Research Center, Toronto, Canada; ' London School of Hygiene and Tropical Medicine, London, United Kingdom

\subsection{6/oemed-2013-101717.264}

Objectives Wood dust is known to be associated with a range of respiratory effects including reduced lung function, increased 